\title{
Evaluación del protocolo fast-track para artroplastia total de rodilla: impacto sobre estancia hospitalaria, visitas al servicio de urgencias e infección.
}

DOI: http//dx.doi.ORG/10.37315/SOTOCAV20212865640

\author{
MOLINA PÉREZ M, SEGARRA MUÑOZ B, CORTÉS TRONCH V, SÁNCHEZ-CORTÉS A, FERNÁNDEZ GARCÍA E.
}

DEPARTAMENTO DE CIRUGÍA ORTOPÉDICA Y TRAUMATOLOGÍA DEL HOSPITAL UNIVERSITARIO DE LA RIBERA. ALZIRA, VALENCIA.

\begin{abstract}
Resumen
Objetivos. Analizar las diferencias en estancia hospitalaria, readmisión e infección en pacientes intervenidos de artroplastia total de rodilla en el periodo previo y posterior a la implantación del protocolo fast-track. Material y métodos. Estudio observacional retrospectivo de cohortes de 777 pacientes. Las variables a estudio fueron: estancia hospitalaria media, readmisión y número de visitas al Servicio de Urgencias y tasa de infección. Resultados. Se obtuvo una muestra homogénea con predominio de mujeres y una edad media de 70 años. Los valores pre y post implantación del protocolo para las variables fueron: estancia hospitalaria 5,1 vs 3,2 días ( $p<0,001$ ), pacientes con readmisión $12,2 \%$ vs $21,9 \%$ $(p<0,001)$, visitas a urgencias 74 vs $118(p<0,001)$. La tasa de infección fue similar en ambos grupos a todos los niveles. Conclusiones. El protocolo fast-track disminuye la estancia hospitalaria y aumenta la tasa de readmisiones. El riesgo de infección no se ve aumentado.
\end{abstract}

\section{Summary}

Objectives: To analyze the differences in length of stay, readmission and infection in patients undergoing total knee arthroplasty before and after the implementation of a fast-track protocol. Material and methods. Retrospective observational follow-up study in 777 patients. The variables under study were: length of stay, readmission and total number of Emergency Department (ED) visits and rate of infection. Results. A homogeneous sample of 777 patients was obtained with women predominance and mean age of 70 years. The values before and after fast-track implantation were: length of stay 5,1 vs 3,2 days $(p<0,001)$, patients underwent readmission $12,2 \%$ vs $21,9 \%(p<0,001)$, ED visits 74 vs 118 ( $p<0,001)$. Rate of infection was similar in both groups for all levels. Conclusions. Fast-track protocols improve length of stay without affecting the rate of infection. Special care should be given to the increased readmission.

Palabras clave: Rodilla, Artroplastia, Fast-Track, Readmisión

\section{Correspondencia:}

Dra. Marta Molina Pérez

Correo electrónico: martamolipe@gmail.com 


\section{INTRODUCCIÓN}

En el año 2010 la Organización Mundial de la Salud consideró la artrosis una de las enfermedades crónicas más invalidantes ${ }^{1}$. Su incidencia se ha visto aumentada $\mathrm{y}$ continúa al alza debido a factores como el envejecimiento, el aumento del IMC o como secuela de cirugías previas. Pese a la existencia de diferentes escalones terapéuticos, la cirugía de artroplastia total de rodilla (ATR) se erige como la solución definitiva, hasta el punto de suponer un reto socio-sanitario para los servicios de Cirugía Ortopédica y Traumatología (COT).

Con la premisa de aliviar la presión asistencial que supone y conseguir una rápida recuperación del paciente nacen los programas fast-track o enhanced recovery after surgery (ERAS). La cirugía fast-track viene definida como un conjunto de actuaciones estandarizadas perioperatorias multidisciplinares, encaminadas a acelerar la recuperación del paciente sin incrementar la morbilidad de los cuidados tradicionales ${ }^{2}$. Dicho de otro modo, la recuperación es facilitada por el enfoque multimodal dirigido a modular de una forma global la respuesta al estrés quirúrgico ${ }^{3}$. Nace en los años 90 y aunque sus beneficios se describen por primera vez en pacientes intervenidos de cirugía de colón mediante laparoscopia ${ }^{4}$, el concepto fue descrito por primera vez por el cirujano danés Henry Kehlet en $1997^{5}$. Kehlet hipotetizó que el estrés quirúrgico inflamatorio y las alteraciones metabólicas y endocrinas asociadas, así como la inmovilización postoperatoria, favorecían la disfunción orgánica, expresada mediante síntomas postoperatorios tan habituales como dolor, náuseas, vómitos o alteraciones cognitivas como el delirium, lo que conduce a un deterioro funcional que implica un mayor ingreso y posterior rehabilitación, determinando el ritmo de recuperación del paciente.

La hipótesis principal de este trabajo es que la cirugía fasttrack aplicada al campo de la artroplastia total de rodilla es capaz de mejorar tanto la calidad de la asistencia prestada, como la satisfacción global del proceso ${ }^{6}$. Con la implantación del programa fast-track, es predecible encontrar una disminución de la estancia hospitalaria ${ }^{7,8}$. Del mismo modo, se ha descrito la posibilidad de que los índices de reingresos por complicaciones no se vean aumentados $^{8,9}$, especialmente aquellos debidos a la infección, complicación quirúrgica más temida por los cirujanos ortopédicos ${ }^{10}$.

El objetivo principal de este estudio es analizar las diferencias tras la implantación del protocolo fast-track en estancia hospitalaria, número de reingresos en los Servicios de Urgencias y riesgo de infección en una muestra de pacientes intervenidos de ATR.

\section{MATERIAL Y MÉTODO}

Se ha llevado a cabo un estudio observacional retrospectivo de cohortes en el servicio de COT del Hospital Universitario de la Ribera. Con la colaboración del servicio de Documentación Clínica se obtuvieron todos los pacientes intervenidos bajo el descriptor "sustitución total de rodilla" para los periodos de tiempo previo a la implantación del protocolo fast-track (desde octubre de 2015 a octubre de 2016) y posterior a la implantación del mismo (desde octubre de 2017 a octubre de 2018). Se seleccionaron pacientes sometidos a artroplastia total de rodilla primaria por gonartrosis, con un seguimiento mínimo de un año. Entre los criterios de exclusión se encontraban pacientes intervenidos de prótesis unicompartimentales, femoropatelares y prótesis de revisión implantadas de forma primaria. Se excluyeron del estudio pacientes pertenecientes a otras áreas de salud para evitar un sesgo durante el seguimiento. El estudio fue aprobado por el Comité de Ética.

Las variables consideradas a estudio se recogieron para cada cohorte. Se han incluido datos sociodemográficos para la caracterización de la muestra. La estancia hospitalaria fue definida como el número de pernoctaciones realizadas en el hospital. Se revisaron los apuntes de evolución (Atención Especializada, Atención Primaria y Urgencias) para estudiar la readmisión hospitalaria, considerándose ésta como una consulta al Servicio de Urgencias Hospitalarias tras el alta. Se estratificó el riesgo de readmisiones por semanas, diferenciándose aquellas que se produjeron en los primeros $7,15,21$ y 30 días post alta. Del mismo modo, se estudió el número de visitas a urgencias total realizadas por los pacientes, dada la potencial reincidencia de algunos de ellos.

Por último, se analizó la variable de infección revisando los apuntes de evolución y el historial de recetas médicas de los pacientes, definiéndose dos tipos. La infección superficial, que incluye la presencia de signos de Celso: rubor, calor, dolor, tumor e impotencia funcional y/o necesidad de tratamiento antibiótico oral o intravenoso. Y la infección profunda, que incluye además de los signos de Celso y necesidad de antibiótico, la necesidad de cirugía, bien mediante lavado articular, cirugía de recambio en un tiempo o cirugía en dos tiempos.

Los datos han sido extraídos en formularios específicamente diseñados y después volcados a una tabla en Microsoft Excel (Microsoft Inc.). Utilizando el software estadístico Statistical Package for the Social Sciences (IBM) se ha realizado un análisis descriptivo y grafico de las principales variables objeto de estudio del trabajo. Para las variables cualitativas se realizó la prueba chi-cuadrado, y para las variables cuantitativas la comparación de medias utilizando el test más apropiado en cada circunstancia. Se ha considerado un valor de $p<0,05$ como estadísticamente significativo.

\section{RESULTADOS}

Se obtuvo una muestra homogénea de 777 pacientes, 370 pertenecientes al periodo pre fast-track y 407 al periodo post fast-track. Las mujeres representan el $68 \%$ del total, siendo mayoritarias en ambas cohortes (Fig. 1). La edad media para ambos grupos fue de 70 años. No se observaron diferencias entre ambos grupos. 


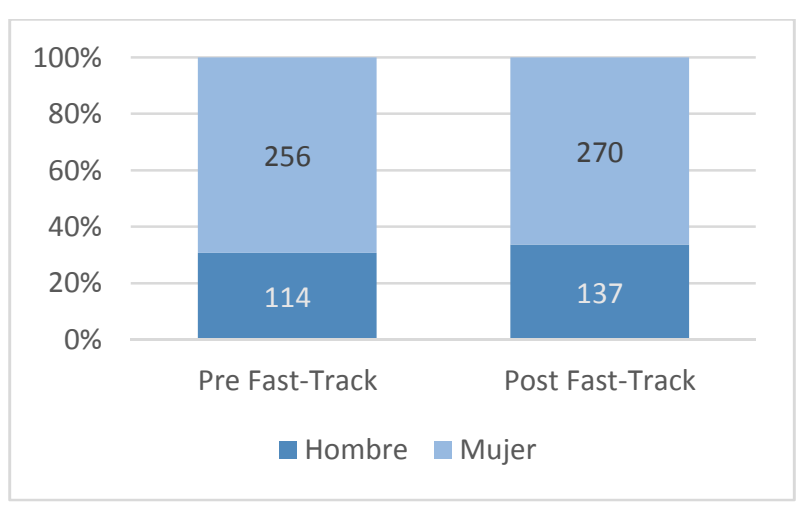

Figura 1. Sexo por cohortes

La estancia media hospitalaria se redujo desde los 5,1 días hasta los 3,2 días tras la implantación del fast-track $(p<0,0001)$.

El porcentaje de pacientes con readmisión hospitalaria al Servicio de Urgencias durante el primer año en el grupo pre fast-track fue del $12 \%$ vs. $22 \%$ para el periodo post fasttrack $(p=0,0001)$. Al estratificar este resultado por semanas, resultó significativo el resultado en aquellos pacientes que consultaron durante los primeros 30 días tras la cirugía siendo superior para el post fast-track (9\% vs $14 \%$; $\mathrm{p}=0,025)$.

El número total de visitas al Servicio de Urgencias para ambos grupos durante un año de seguimiento fue de 192, correspondiéndose 74 (39\%) al periodo pre y $118(62 \%)$ al periodo post. Además, desglosándose por semanas, este resultado fue significativo para los primeros 21 y 30 días tras la cirugía, con un p-valor respectivo de 0,036 y $<0,0001$. El principal motivo de consulta fue el dolor postquirúrgico (Fig. 2).

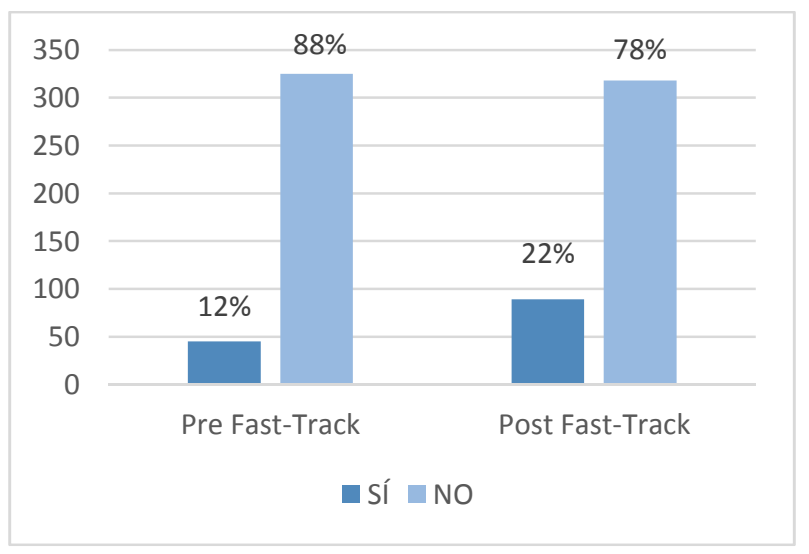

\begin{tabular}{|l|l|c|c|}
\hline & & & \\
\hline & Recuento & 318 & 81 \\
\hline & $\%$ & $78,1 \%$ & $21,9 \%$ \\
\hline PRE & Recuento & 325 & 45 \\
\hline & $\%$ & $87,8 \%$ & $12,2 \%$ \\
\hline
\end{tabular}

Figura 2. Porcentaje de pacientes readmitidos en el Servicio de Urgencias.

La infección global, sumatorio de la superficial y profunda fue ligeramente mayor para el grupo pre fast-track sin alcanzar significación estadística $(8,4 \%$ vs. $8,1 \%)$. Tampoco se encontraron diferencias al desagregar los datos en infección superficial y profunda (Fig. 3).

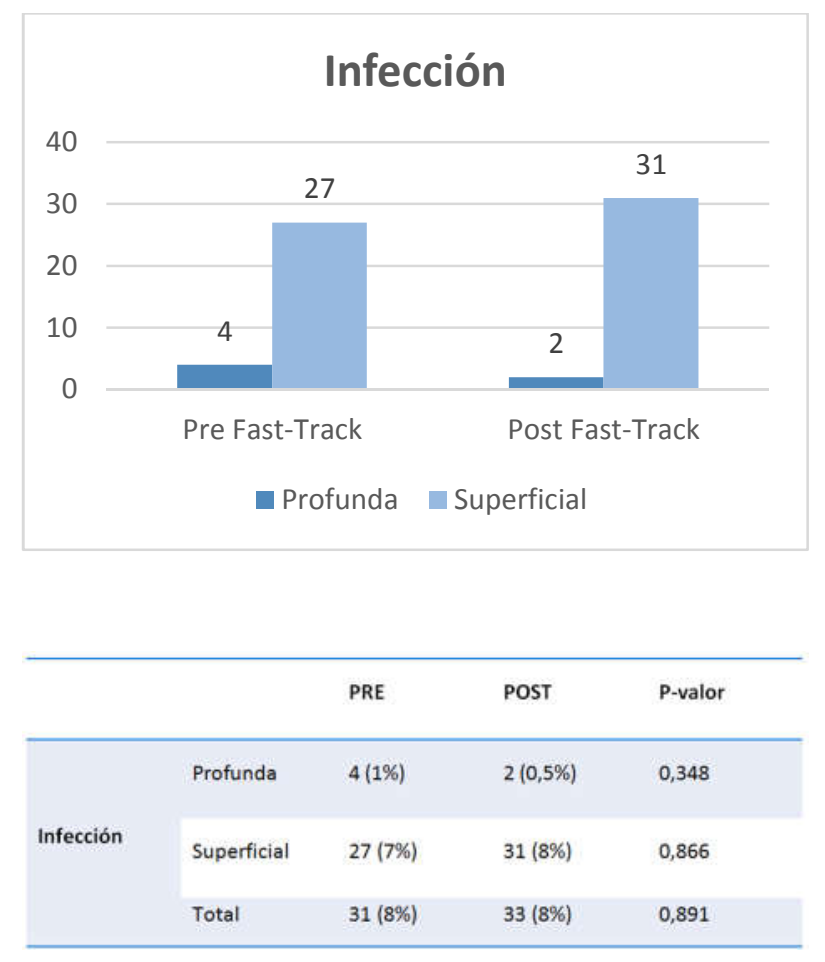

Figura 3. Tasa de infección superficial, Profunda y Total por grupos. No se alcanza significación. 


\begin{tabular}{|c|c|c|c|c|}
\hline \multicolumn{5}{|c|}{ Resultados de la muestra } \\
\hline \multicolumn{2}{|c|}{ Variable } & $\begin{array}{l}\text { Pre Fast-Track } \\
\quad(n=370)\end{array}$ & $\begin{array}{c}\text { Post Fast-Track } \\
(n=407)\end{array}$ & P-valor \\
\hline \multirow{2}{*}{ Sexo } & Hombres & $114(31 \%)$ & 137 (34\%) & \multirow{2}{*}{0,396} \\
\hline & Mujeres & $256(69 \%)$ & $270(66 \%)$ & \\
\hline \multirow{2}{*}{ Lateralidad } & Derecha & $196(53 \%)$ & 220 (54\%) & \multirow{2}{*}{0,763} \\
\hline & Izquierda & $174(47 \%)$ & 187 (46\%) & \\
\hline \multicolumn{2}{|l|}{ Edad } & $71 \pm 8 / 72 *$ & $70 \pm 8 / 72 *$ & 0,649 \\
\hline \multicolumn{2}{|c|}{$\begin{array}{l}\text { Estancia hospitalaria } \\
\text { (días) }\end{array}$} & $5,1 \pm 1 / 5^{*}$ & $3,2 \pm 1 / 3^{*}$ & $<0,0001$ \\
\hline \multirow{3}{*}{ Infección } & Profunda & $4(1 \%)$ & $2(0,5 \%)$ & 0,348 \\
\hline & Superficial & $27(7 \%)$ & $31(8 \%)$ & 0,866 \\
\hline & Total & $31(8 \%)$ & $33(8 \%)$ & 0,891 \\
\hline
\end{tabular}

\begin{tabular}{|c|c|}
\hline \multicolumn{2}{|c|}{ Protocolo analgésico durante la hospitalización } \\
\hline Medicación & Pauta \\
\hline Oxicodona/Naloxona Clorhidrato & $10 \mathrm{mg} / 12 \mathrm{~h}$ \\
\hline Paracetamol & $1 \mathrm{~g} / 6 \mathrm{~h}$ \\
\hline Dexketoprofeno & $25 \mathrm{mg} / 8 \mathrm{~h}$ \\
\hline Metamizol IV & $2 \mathrm{~g} / 8 \mathrm{~h}$ (de rescate) \\
\hline
\end{tabular}




\section{DISCUSIÓN}

Se ha diseñado un estudio comparativo retrospectivo entre pacientes tratados mediante el protocolo fast-track $y$ aquellos tratados previamente a la implantación del mismo. Ambos subgrupos han demostrado poseer unas características homogéneas, lo que ha permitido demostrar las potenciales ventajas de la implantación de un protocolo fast-track para artroplastia total de rodilla.

Este estudio ha presentado algunas limitaciones. La primera de ellas son las comorbilidades que pueda haber presentado la muestra, pues no han sido consideradas, ni se han recogido los datos de las mismas, lo que puede suponer un sesgo a la hora de analizar las variables. Del mismo modo, la muestra también se ha podido ver influenciada por condicionantes de los propios pacientes. Es posible que algunos de ellos hayan presentado alguna patología grave de nuevo diagnóstico durante el preoperatorio y por tanto hayan sido finalmente excluidos de la lista de espera quirúrgica y no consten en el estudio. Otra limitación se relaciona con el seguimiento del paciente, pues solo se han revisado las anotaciones (Hospitalización, Consulta Externa o Atención Primaria) generadas en el área de influencia del propio hospital y no aquellas generadas en pacientes, que, tras la cirugía hayan precisado asistencia y, por motivo de movilidad o urgencia, hayan sido atendidos en otros hospitales. También han podido existir sesgos en la caracterización de la infección. Se decidió emplear los signos de Celso, reflejados como tales en la historia clínica, y se consideró que una prescripción antibiótica era criterio de infección superficial, pues se entendió que de haber sido descartada la infección con total seguridad, no habría sido prescrito el antibiótico. Dicho esto, es probable que se haya sobreestimado el efecto de la infección en el seguimiento de los pacientes, especialmente cuando la prescripción antibiótica ocurrida en las consultas de Atención Primaria. Dado que los pacientes eran dados de alta de forma precoz, es posible que los médicos de familia se hayan enfrentado a situaciones poco comunes antes, como la evolución de hematomas, que fácilmente pueden haberse confundido con infecciones superficiales.

Nuestra muestra redujo en dos días la estancia media hospitalaria, siguiendo la línea de estudios ya publicados en los países nórdicos, pioneros en la implantación de estos protocolos ${ }^{11}$. La reducción en la estancia media hospitalaria puede explicarse por la combinación de factores tan importantes como el manejo adecuado del dolor, la rehabilitación precoz, la prevención del mareo por pérdida de sangre y la ausencia de necesidad de transfusión ${ }^{12}$. Del mismo modo, en aras de reducir aún más la estancia media hospitalaria, existen posibles líneas de investigación futuras, como contar con plantas de hospitalización específicas con personal formado, la reserva de camas para los pacientes quirúrgicos, así como, intentar que los pacientes sean intervenidos durante los primeros días de la semana, ya que parece que los pacientes intervenidos del jueves en adelante sufren más dificultades para recibir un alta temprana ${ }^{13}$.La muestra estudiada presenta una tasa de infección similar, tanto a nivel superficial como profundo, en ambas cohortes. Esto debe ser visto de forma muy positiva, dado que la infección representa una complicación grave en los pacientes intervenidos de ATR y son muchas las actuaciones desarrolladas a fin de evitar este evento. Con una tasa de infección del 8 '1\% se mantiene la tendencia con otras publicaciones que apoyan la ausencia del aumento en la tasa de complicaciones ${ }^{11}$.

Sin embargo, el protocolo fast-track en nuestra muestra ha demostrado un aumento en las readmisiones hospitalarias, tanto en el porcentaje de pacientes que visitan el Servicio de Urgencias (de hasta un 10\% Urgencias el primer año y de un $5 \%$ durante el primer mes), como en el número total de visitas al mismo. El motivo principal de readmisión fue el dolor. Esto ha motivado el desarrollo de nuevos protocolos de analgesia en nuestro hospital gracias a la colaboración de la Unidad del Dolor. Pese a la ausencia de estudios analíticos actualizados, la impresión global de los facultativos ha sido muy positiva, pues se observa una menor tasa de readmisiones por este motivo desde la implantación de dicho nuevo protocolo.

El principal alcance del presente trabajo es demostrar la efectividad de las medidas puestas en marcha y allanar el camino para la implantación de mejoras constantes. Como se ha descrito, esta es la esencia de la creación de un protocolo fast-track: un grupo de trabajo dedicado que se encargue de definir nuevas metas y líneas de investigación para la mejora de los resultados clínicos y organizativos. Considerando que los avances en medicina van de la mano de las nuevas tecnologías, una buena línea de investigación podría dedicarse a estudiar la elaboración y desarrollo de programas de rehabilitación online con videos explicativos a los que acceder desde el domicilio, evitando costes de desplazamiento. De igual modo, sería conveniente evaluar la percepción del protocolo fast-track por parte de los pacientes, así como la perspectiva desde el lado del profesional. La propuesta consistiría en elaborar encuestas de satisfacción dirigidas a los sujetos y evaluar la percepción subjetiva del proceso.

Todas las medidas comentadas anteriormente suponen un ahorro en costes para la institución, algo que, sin haberse probado en este estudio, ni ser objetivo primordial de la implantación de protocolos fast-track, puede intuirse como ya han demostrado otros estudios llevados a cabo en España $^{14}$.

En conclusión, los Servicios de COT deben conocer la existencia de los protocolos fast-track para ATR como respuesta al reto sociosanitario planteado y deberían fomentar su aplicación y desarrollo ya que mejoran la calidad asistencial, reduciendo la estancia media hospitalaria sin afectar a la tasa de infección. Sin embargo, debe prestarse especial cuidado a la evolución temprana de los pacientes y sus potenciales necesidades para evitar las readmisiones a los Servicios de Urgencias por motivos como el dolor postquirúrgico. El ahorro que supone la implantación de estos protocolos debe ser estudiado más a fondo. Son necesarios más estudios considerando las comorbilidades del paciente, para distinguir así, aquellos que obtendrían un máximo beneficio de aquellos que incluso podrían verse perjudicados. 


\section{Bibliografía}

1. Hoy DG, Smith E, Cross M, Sanchez-Riera L, Buchbinder R, Blyth FM, et al. The global burden of musculoskeletal conditions for 2010: an overview of methods. Ann Rheum Dis. 2014; 73(6):982-9. doi: 10.1136/annrheumdis-2013-204344. Epub 2014 Feb 18. PMID: 24550172.

2. Fearon KC, Ljungqvist $\mathrm{O}$, Von Meyenfeldt $\mathbf{M}$, Revhaug $\mathbf{A}$, Dejong $\mathbf{C H}$, Lassen $\mathrm{K}$, et al. Enhanced recovery after surgery: a consensus review of clinical care for patients undergoing colonic resection. Clin Nutr. 2005; 24(3):466-77. doi: 10.1016/j.clnu.2005.02.002. Epub 2005 Apr 21. PMID: 15896435.

3. Soffin EM, YaDeau JT. Enhanced recovery after surgery for primary hip and knee arthroplasty: a review of the evidence. Br $\mathrm{J}$ Anaesth. 2016; 117(suppl 3):iii62-iii72. doi: 10.1093/bja/aew362. PMID: 27940457.

4. Bardram L, Funch-Jensen P, Jensen P, Crawford ME, Kehlet H. Recovery after laparoscopic colonic surgery with epidural analgesia, and early oral nutrition and mobilisation. Lancet. 1995; 345(8952):763-4. doi: 10.1016/s0140-6736(95)90643-6. PMID: 7891489.

5. Kehlet H. Multimodal approach to control postoperative pathophysiology and rehabilitation. Br J Anaesth. 1997; 78(5):606-17. doi: 10.1093/bja/78.5.606. PMID: 9175983.

6. Kehlet H, Wilmore DW. Evidence-based surgical care and the evolution of fast-track surgery. Ann Surg. 2008; 248(2):189-98. doi: 10.1097/SLA.0b013e31817f2c1a. PMID: 18650627.

7. Raphael M, Jaeger M, van Vlymen J. Easily adoptable total joint arthroplasty program allows discharge home in two days. Can J Anaesth. 2011; 58(10):902-10. doi: 10.1007/s12630-011-9565-8. Epub 2011 Aug 6. PMID: 21822756.

8. Deng QF, Gu HY, Peng WY, Zhang Q, Huang ZD, Zhang C, Yu YX. Impact of enhanced recovery after surgery on postoperative recovery after joint arthroplasty: results from a systematic review and meta-analysis. Postgrad Med J. 2018; 94(1118):678-693. doi: 10.1136/postgradmedj-2018-136166. Epub 2019 Jan 21. PMID: 30665908.

9. Glassou EN, Pedersen AB, Hansen TB. Risk of re-admission, reoperation, and mortality within 90 days of total hip and knee arthroplasty in fast-track departments in Denmark from 2005 to 2011. Acta Orthop. 2014; 85(5):493-500. doi: 10.3109/17453674.2014.942586. Epub 2014 Jul 18. PMID: 25036718; PMCID: PMC4164867.

10. Molko S, Dasí-Sola M, Marco F, Combalia A. Clinical practices for primary hip and knee arthroplasties in Spain: A national study. Rev Esp Cir Ortop Traumatol (Engl Ed). 2019; 63(6):408-415. English, Spanish. doi: 10.1016/j.recot.2019.06.005. Epub 2019 Sep 23. PMID: 31558430.

11. Petersen PB, Jørgensen CC, Kehlet H. Lundbeck Foundation Center for Fast-track Hip and Knee Replacement collaborative group. Temporal trends in length of stay and readmissions after fast-track hip and knee arthroplasty. Dan Med J. 2019l; 66(7):A5553. PMID: 31256778.

12. Husted H, Lunn TH, Troelsen A, Gaarn-Larsen L, Kristensen BB, Kehlet $\mathbf{H}$. Why still in hospital after fast-track hip and knee arthroplasty? Acta Orthop. 2011; 82(6):679-84. doi: 10.3109/17453674.2011.636682. Epub 2011 Nov 9. PMID: 22066560; PMCID: PMC3247885.

13. Mathijssen NM, Verburg H, van Leeuwen CC, Molenaar TL, Hannink G. Factors influencing length of hospital stay after primary total knee arthroplasty in a fast-track setting. Knee Surg Sports Traumatol Arthrosc. 2016; 24(8):2692-6. doi: 10.1007/s00167-015-3932-x. Epub 2015 Dec 19. PMID: 26685683.

14. Wilches C, Sulbarán JD, Fernández JE, Gisbert JM, Bausili JM, Pelfort X. Fast-track recovery technique applied to primary total hip and knee replacement surgery. Analysis of costs and complications. Rev Esp Cir Ortop Traumatol. 2017; 61(2):111-116. English, Spanish. doi: 10.1016/j.recot.2016.10.002. Epub 2017 Jan 8. PMID: 28073671. 